Pacific

Journal of

Mathematics

APPLICATION OF RESTRICTION OF FOURIER TRANSFORMS TO AN EXAMPLE FROM REPRESENTATION THEORY

L. Barchini and Mark R. Sepanski 


\title{
APPLICATION OF RESTRICTION OF FOURIER TRANSFORMS TO AN EXAMPLE FROM REPRESENTATION THEORY
}

\author{
L. BARChini And Mark R. SEPanski
}

\begin{abstract}
This paper uses restriction of Fourier transforms to construct explicit realizations of certain irreducible unitary representations of $S U(n, n)$. The realizations begin with generalizations of the classical Szegö map. Boundary values of these Szegö maps naturally lead to certain restrictions of Fourier transforms. The image of these restrictions provide concrete constructions of unitary representations as $L^{2}$ spaces on certain orbits. The $S U(n, n)$ invariance of the $L^{2}$ spaces and inner products follows immediately from the restriction maps and the natural pairing between certain degenerate principal series.
\end{abstract}

\section{Introduction.}

Calculating explicit and natural constructions of unitary representationsespecially singular ones-has been a very fruitful field of study in representation theory. Consider, for instance, the Metaplectic representation and its many applications. One of the reasons explicit realizations are so useful is because detailed knowledge of a representation frequently comes through use of a good realization. This paper studies and constructs a number of explicit realizations for certain unitary representations of $S U(n, n)$. The central technique employs certain restrictions of Fourier transforms $([\mathbf{1 5}])$ that arise naturally in the study of the representation theory of $S U(n, n)$. Our approach is different than the one usually adopted in such studies of this kind (e.g., [8], [12], [13], [14]) where "extensions" of Fourier transforms are mainly used.

In our approach, the representations naturally arise from an examination of various Szegö maps and their boundary values which immediately lead to certain restrictions of Fourier transforms. There are several advantages to this line of study. The first is that the invariance of our spaces and inner products are very natural from this point of view. The second is that precise knowledge of the $K$-types is not needed. The third is that the techniques employed are independent of a multiplicity one assumption on $K$-types. 
To be more specific about the results of this study, write $G=S U(n, n)$, $K=S(U(n) \times U(n))$, and $G / P$ for the closed $G$-orbit in the boundary of $G / K$. The unbounded realization of $G / K$ may be identified with $\mathcal{D}^{+}=$ $H+i H^{+}$where $H$ and $H^{+}$are the set of $n \times n$ Hermitian matrices and $n \times n$ positive definite Hermitian matrices, respectively. More generally, the semi-definite $G$-orbits of $G_{\mathbb{C}} / P_{\mathbb{C}}$ may be described on an open dense set as $H+i \mathcal{O}_{p}$ where

$$
\mathcal{O}_{p}=\{X \in H \mid \text { signature of } X \text { is }(p, 0)\} .
$$

$\mathcal{O}_{p}$ is an orbit under the action of the Levi part of $P$ and comes equipped with a uniquely defined equivariant measure, $d \mu_{p}$. Then the main application of our study of the restriction of the Fourier transform shows that

$$
L^{2}\left(\mathcal{O}_{p}, d \mu_{p}\right)
$$

is an irreducible unitary representation of $S U(n, n)$ (Theorem 10.4). Though this statement is already known $([\mathbf{1 2}])$, we believe the techniques in our new approach yield a more complete understanding of this representation. We also expect the same techniques to be applicable to a wider family of representations-at least including the representations associated to certain orbits in real semisimple Jordan algebras ([13]).

In more detail, we begin with certain pairs of degenerate principal series on $G / P$. For certain parameters, depending on each choice of $\mathcal{O}_{p}$, the appropriate principal series may be realized in the noncompact picture as $L^{2}\left(H, \operatorname{det}\left(I+X^{2}\right)^{ \pm(n-p)} d X\right)$ and is denoted by $L^{2}(H)^{ \pm}$, respectively. Using techniques similar to [11] and [1], we write down a $S z e g \ddot{o}$ map, $S: L^{2}(H)^{+} \rightarrow C^{\infty}\left(\mathcal{D}^{+}\right)$. It turns out that $S$ acts on a function $f \in L^{2}(H)^{+}$by the particularly easy formula

$$
S f(\eta)=\int_{H} \operatorname{det}(X-\eta)^{-p} f(X) d X
$$

for each $\eta \in \mathcal{D}^{+}$(Theorem 5.3). Writing $B$ for the boundary value map taking $\mathcal{D}^{+}$to $H$, it is possible to form a commutative diagram defining an intertwining map, $A: L^{2}(H)^{+} \rightarrow L^{2}(H)^{-}$, of the form

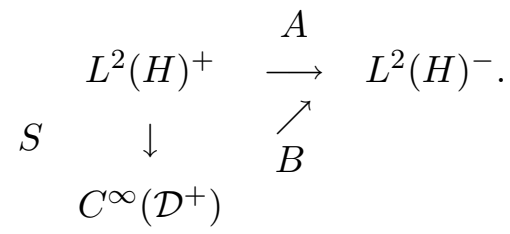

For functions $\phi \in \mathcal{S}(H)$, the Schwartz functions on $H$, it is possible to see that the action of $A$ may be rewritten as

$$
A \phi(X)=i^{n p} \int_{\mathcal{O}_{p}} \mathrm{e}^{i \operatorname{tr}(X \xi)} \check{\phi}(\xi) d \mu_{p}(\xi)
$$


where $\check{\phi}$ is the inverse Fourier transform of $\phi$ on $H$ (Theorem 7.2). Equation (1.3) suggests a second splitting of the singular integral defining $A$. Namely, consider the two maps $F_{R}: L^{2}(H)^{+} \rightarrow L^{2}\left(\mathcal{O}_{p}, d \mu_{p}\right)$ and $F_{E}$ : $L^{2}\left(\mathcal{O}_{p}, d \mu_{p}\right) \rightarrow L^{2}(H)^{-}$given by

$$
F_{R} \phi=\left.\check{\phi}\right|_{\mathcal{O}_{p}}
$$

when $\phi \in \mathcal{S}(H)$, where $\left.\check{\phi}\right|_{\mathcal{O}_{p}}$ denotes restriction of $\check{\phi}$ to $\mathcal{O}_{p}$, and

$$
F_{E} \psi(X)=i^{n p} \int_{\mathcal{O}_{p}} \mathrm{e}^{i \operatorname{tr}(\xi X)} \psi(\xi) d \mu_{p}(\xi)
$$

for $\psi \in \operatorname{Im}\left(F_{R}\right)$. The first is a restriction of the Fourier transform and the second is the more usual "extension" of the Fourier transform. These maps are proved to be continuous (Theorem 9.1) and yield the commutative diagram

$$
\begin{array}{cccc}
L^{2}(H)^{+} & \stackrel{A}{\longrightarrow} & L^{2}(H)^{-} & \\
& \searrow & \uparrow & F_{E} . \\
& F_{R} & L^{2}\left(\mathcal{O}_{p}, d \mu_{p}\right) &
\end{array}
$$

This diagram is used to make $L^{2}\left(\mathcal{O}_{p}, d \mu_{p}\right)$ into a representation of $G$ by requiring all maps to be $G$-maps (Theorem 10.2).

The point of working with $L^{2}\left(\mathcal{O}_{p}, d \mu_{p}\right)$ is that it comes equipped with its own inner product denoted by

$$
\left\langle f_{1}, f_{2}\right\rangle_{\mathcal{O}_{p}}=\int_{\mathcal{O}_{p}} f_{1}(\xi) \overline{f_{2}(\xi)} d \mu_{p}(\xi)
$$

for $f, g \in L^{2}\left(\mathcal{O}_{p}, d \mu_{p}\right)$. In fact, it is proved that this structure makes $L^{2}\left(\mathcal{O}_{p}, d \mu_{p}\right)$ into a irreducible unitary representation (Theorem 10.4) of $G$. The key to seeing the invariance of the inner product is to relate it to an invariant form on $L^{2}(H)^{+}$. Indeed, consider the natural $G$-invariant pairing of $L^{2}(H)^{+}$and $L^{2}(H)^{-}$given by

$$
\langle f, g\rangle_{A}=\int_{H} f(X) \overline{A g(X)} d X
$$

for $f, g \in L^{2}(H)^{+}$. The central identity fitting everything together is

$$
\langle f, g\rangle_{A}=\left\langle F_{R} f, F_{R} g\right\rangle_{\mathcal{O}_{p}}
$$

(Theorem 10.3). Thus the $G$-invariance of the $L^{2}$ inner product follows directly and immediately.

Finally, denoting the kernel of $R_{F}$ as $\mathcal{K}$ (which is the same as the kernel of $\left.\langle\cdot, \cdot\rangle_{A}\right), R_{F}$ therefore induces a bijective intertwining isometry between the completion of $L^{2}(H)^{+} / \mathcal{K}$ and $L^{2}\left(\mathcal{O}_{p}, d \mu_{p}\right)$. 
The authors thank A. Korányi, R. Kunze, A. Noell, D. Ullrich, and R. Zierau for many insightful conversations on this project.

\section{Preliminaries.}

Let $G=S U(n, n)$. Breaking up the $2 n \times 2 n$ matrices into four $n \times n$ blocks, write:

$$
J=\left(\begin{array}{cc}
0 & -i I_{n} \\
i I_{n} & 0
\end{array}\right)
$$

Unless noted otherwise, we will use the following realization throughout the paper:

$$
G=\left\{g \in S L(2 n, \mathbb{C}) \mid g^{*} J g=J\right\} .
$$

It is useful to gather a few simple facts about $G$ for later use.

Lemma 2.1. Write the $2 n \times 2 n$ matrix $g=\left(\begin{array}{cc}A & B \\ C & D\end{array}\right)$ as four $n \times n$ blocks.

1) $G$ consists of the matrices $g \in S L(2 n, \mathbb{C})$ satisfying

$$
A^{*} C=C^{*} A, \quad D^{*} B=B^{*} D, \quad A^{*} D-C^{*} B=I .
$$

2) $G$ consists of the matrices $g \in S L(2 n, \mathbb{C})$ satisfying

$$
A B^{*}=B A^{*}, C D^{*}=D C^{*}, A D^{*}-B C^{*}=I .
$$

3) For $g \in G$,

$$
g^{-1}=\left(\begin{array}{cc}
D^{*} & -B^{*} \\
-C^{*} & A^{*}
\end{array}\right)
$$

4) $K=\{g \in G \mid A=D, B=-C\}$ is a maximally compact subgroup of $G$. $K$ may also be described as

$$
K=\left\{\left(\begin{array}{cc}
A & B \\
-B & A
\end{array}\right) \mid(A+i B, A-i B) \in S(U(n) \times U(n))\right\} .
$$

5) $P=\{g \in G \mid C=0\}$ and $\bar{P}=\{g \in G \mid B=0\}$ are parabolic subgroups of $G$. $P$ can also be described as

$$
\begin{array}{r}
P=\left\{\left(\begin{array}{cc}
A & B \\
0 & A^{*-1}
\end{array}\right) \mid A \in G L(n, \mathbb{C}), \operatorname{det}(A) \in \mathbb{R}^{\times}\right. \\
\left.B \in \mathfrak{g l}(n, \mathbb{C}), A^{-1} B=\left(A^{-1} B\right)^{*}\right\} .
\end{array}
$$


6) $P$ admits a Langlands decomposition $P=L N$ with $L=M A$ where

$$
\begin{array}{r}
M=\left\{\left(\begin{array}{cc}
A & 0 \\
0 & A^{*-1}
\end{array}\right) \mid A \in G L(n, \mathbb{C}), \operatorname{det}(A)= \pm 1\right\}, \\
A=\left\{\left(\begin{array}{cc}
a I & 0 \\
0 & a^{-1} I
\end{array}\right) \mid a \in \mathbb{R}^{>0}\right\}, \\
N=\left\{\left(\begin{array}{cc}
I & X \\
0 & I
\end{array}\right) \mid X^{*}=X\right\} .
\end{array}
$$

Likewise, $\bar{P}=L \bar{N}$ where

$$
\bar{N}=\left\{\left(\begin{array}{cc}
I & 0 \\
X & I
\end{array}\right) \mid X^{*}=X\right\} .
$$

The the representations of $G$ to be studied will be induced from the following characters.

Definition 2.1. Let $p \in \mathbb{Z}, 0 \leq p \leq n$.

1) The character $\chi_{p}: K \rightarrow S^{1}$ acts by

$$
\chi_{p}\left(\begin{array}{cc}
A & B \\
-B & A
\end{array}\right)=\operatorname{det}(A+i B)^{p} .
$$

2) The character $\delta_{p}: M \rightarrow\{ \pm 1\}$ acts by

$$
\delta_{p}\left(\begin{array}{cc}
A & 0 \\
0 & A^{*-1}
\end{array}\right)=\operatorname{det}(A)^{p} .
$$

3) Write $\mathfrak{a}_{0}=\operatorname{Lie}(A)$. Let $\epsilon: \mathfrak{a}_{0} \rightarrow \mathbb{R}$ by

$$
\epsilon\left(\begin{array}{cc}
a I & 0 \\
0 & -a I
\end{array}\right)=a
$$

and $\nu_{p}: \mathfrak{a}_{0} \rightarrow \mathbb{R}$ by

$$
\nu_{p}=n(n-p) \epsilon
$$

(the differential of the character $\operatorname{det}(A)^{n-p}$ ).

4) Write the Cartan decomposition for $\operatorname{Lie}(G)$ as $\operatorname{Lie}(G)=\operatorname{Lie}(K)+\mathfrak{p}$, write $\mathfrak{a}_{\mathfrak{p}}$ for the maximal Abelian subalgebra of $\mathfrak{p}$ consisting of diagonal matrices, and $\rho: \mathfrak{a}_{\mathfrak{p}} \rightarrow \mathbb{R}$ for the half sum of restricted weights. An easy calculation shows $\left.\rho\right|_{\mathfrak{a}_{0}}=n^{2} \epsilon$.

Explicitly, we will study the degenerate principal series induced from the characters $\delta_{p} \otimes \pm \nu_{p}$ of the maximal parabolic $P$.

Definition 2.2. Let

$$
I_{p}^{ \pm}=\operatorname{Ind}_{P}^{G}\left(\delta_{p} \pm \nu_{p}\right)
$$

(smooth, normalized induction). 
In other words,

$(2.5) I_{p}^{ \pm}=\left\{f: G \rightarrow \mathbb{C}\right.$, smooth $\left.\mid f(x \operatorname{man})=\delta_{p}^{-1}(m) \mathrm{e}^{-\left( \pm \nu_{p}+\rho\right) \log (a)} f(x)\right\}$ with a $G$ action of

$$
g f(x)=f\left(g^{-1} x\right) .
$$

Beginning in Section 5, we will also make use of the noncompact picture of these induced representations $([\mathbf{9}], \S 7.1)$. To that purpose, decompose $G$ as $K M A N$ and write the $A$ part of $g$ as $\mathrm{e}^{H(g)}$. Then the associated Hilbert space for $I_{p}^{ \pm}$is

$$
L^{2}\left(\bar{N}, \mathrm{e}^{ \pm 2 \operatorname{Re} \nu_{p} H(\bar{n})} d \bar{n}\right)
$$

where $d \bar{n}$ is Haar measure.

Definition 2.3. Let $X, Y \in \mathfrak{g l}(n, \mathbb{C})$.

1) Given a fixed presentation $Z=X+i Y$, define

$$
\begin{gathered}
\bar{Z}=X-i Y, \\
Z^{*}=X^{*}+i Y^{*}
\end{gathered}
$$

so that $\bar{Z}^{*}$ is the normal transpose complex conjugation.

2) Given $g=\left(\begin{array}{cc}A & B \\ C & D\end{array}\right) \in \mathfrak{g l}(2 n, \mathbb{C})$, let

$$
\eta_{g}=(C+i D)(A+i B)^{-1}
$$

which is well defined for almost all $g$ and let

$$
\alpha_{g}=A+i B \text {. }
$$

3) Write $H=H(n)$ for the set of $n \times n$ Hermitian matrices,

$$
H=\left\{X \in \mathfrak{g l}(n, \mathbb{C}) \mid X^{*}=X\right\},
$$

and $H^{ \pm}$for the positive, respectively negative, definite ones,

$$
H^{ \pm}=\{X \in H \mid \pm X>0\} \text {. }
$$

4) Write

$$
\begin{array}{r}
\mathcal{D}=\{X+i Y \mid X, Y \in H\}, \\
\mathcal{D}^{+}=\left\{X+i Y \mid X \in H, Y \in H^{+}\right\} \\
\mathcal{D}^{-}=\left\{X+i Y \mid X \in H, Y \in H^{-}\right\} .
\end{array}
$$

It is easy to check the following.

Lemma 2.2. The mapping

$$
g \rightarrow \eta_{g}
$$

implements an isomorphism between $G / K$ and $\mathcal{D}^{+}$. In particular, $\eta_{g}^{*}=\eta_{g}$ and $\alpha_{g}$ is invertible. 


\section{The Szegö Map to Sections on $G / K$.}

Definition 3.1. Write $C^{\infty}\left(G / K, \chi_{p}\right)$ for the smooth sections on $G / K$ of the line bundle induced by $\chi_{p}$. We will view this as

$$
\left\{f: G \rightarrow \mathbb{C}, \text { smooth } \mid f(g k)=\chi_{p}^{-1}(k) f(g) \forall g \in G, k \in K\right\} .
$$

The central tool used to analyze the representations in this paper is the following Szegö map.

Definition 3.2. Define the Szegö map,

$$
S: I_{p}^{+} \rightarrow C^{\infty}\left(G / K, \chi_{p}\right),
$$

to be the $G$-intertwining operator mapping $f \rightarrow S f$ given by

$$
S f(g)=\frac{1}{\operatorname{Vol}(M \bigcap K)} \int_{K} f(g k) \chi_{p}(k) d k .
$$

In the following, we show that the map $S$ is a kernel operator. This will permit us to switch to the noncompact picture for $I_{p}^{+}$and identify $C^{\infty}\left(G / K, \chi_{p}\right)$ as the set of smooth functions on the tube domain $\mathcal{D}^{+}$.

The first step is to rewrite $S$ as an integral over $\bar{N}$. As usual, given $g \in G$, decompose $g$ according to

$$
G=K \exp \left(\mathfrak{m}_{0} \cap \mathfrak{p}\right) A N
$$

where $\mathfrak{m}_{0}=\operatorname{Lie}(M)$. Therefore write $g=K(g) M(g) A(g) N(g), A(g)=$ $\mathrm{e}^{H(g)}$, and $L(g)=M(g) A(g)$. For future reference, observe $\left.\delta_{p}\right|_{\exp \left(\mathfrak{m}_{0}\right)}=1$ since $\delta_{p}$ is trivial on the connected component of $M$.

Theorem 3.1. For $f \in I_{p}^{+}$,

$$
S f(g)=\int_{\bar{N}} \mathrm{e}^{\left(\nu_{p}-\rho\right) H\left(g^{-1} \bar{n}\right)} \chi_{p}\left(K\left(g^{-1} \bar{n}\right)\right) f(\bar{n}) d \bar{n} .
$$

Proof. This is a standard change of variables. For instance, see [11].

Lemma 3.2. Let $g=\left(\begin{array}{cc}A & B \\ C & D\end{array}\right) \in G, \bar{n}=\left(\begin{array}{cc}I & 0 \\ X & I\end{array}\right) \in \bar{N}$, and $l=$ $\left(\begin{array}{cc}A_{1} & 0 \\ 0 & A_{1}^{*-1}\end{array}\right) \in L . \quad$ Let $\eta=\eta_{g}$ and $\alpha=\alpha_{g}$.

1) Then

$$
\mathrm{e}^{2 n \epsilon H\left(g^{-1} \bar{n} l\right)}=\operatorname{det}\left(A_{1}^{*} A_{1}\right) \operatorname{det}\left(\alpha^{*} \bar{\alpha}\right) \operatorname{det}(X-\eta) \operatorname{det}(X-\bar{\eta}) .
$$

2) Both $\operatorname{det}\left(\alpha^{*} \bar{\alpha}\right)$ and $\operatorname{det}(X-\eta) \operatorname{det}(X-\bar{\eta})$ are in $\mathbb{R}^{\geq 0}$.

3) As a special case,

$$
\mathrm{e}^{n \epsilon H(\bar{n})}=\operatorname{det}\left(I+X^{2}\right)^{\frac{1}{2}} .
$$


4) Finally,

$$
\chi_{1}\left(K\left(g^{-1} \bar{n} l\right)\right)=i^{-n} \operatorname{det}\left(A_{1}\right) \operatorname{det}\left(\bar{\alpha}^{*}\right) \operatorname{det}(X-\bar{\eta}) \mathrm{e}^{-n \epsilon H\left(g^{-1} \bar{n} l\right)} .
$$

Proof.

Set $x=g^{-1} \bar{n} l$ and write $x=K(x) M(x) A(x) N(x)$. Let $g=\left(\begin{array}{cc}A & B \\ C & D\end{array}\right)$, $A(x)=\left(\begin{array}{cc}R_{1} & 0 \\ 0 & R_{2}\end{array}\right)$, and $L(x)=M(x) A(x)=\left(\begin{array}{cc}L_{1} & 0 \\ 0 & L_{2}\end{array}\right)$. Then

$$
\mathrm{e}^{2 n \epsilon H(x)}=\operatorname{det}\left(R_{1}\right)^{2}=\left|\operatorname{det}\left(L_{1}\right)\right|^{2} .
$$

On the other hand, if we write $x=\left(\begin{array}{ll}x_{1} & x_{2} \\ x_{3} & x_{4}\end{array}\right)$ and calculate $x^{*} x$ and compare the $K(x) L(x) N(x)$ expansion to straightforward multiplication, the upper left hand corner yields the equality

$$
L_{1}^{*} L_{1}=x_{1}^{*} x_{1}+x_{3}^{*} x_{3} .
$$

But now Equation (2.3) allows us to calculate $x=g^{-1} \bar{n} l$ and so compute that $x_{1}=\left(D^{*}-B^{*} X\right) A_{1}$ and $x_{3}=\left(-C^{*}+A^{*} X\right) A_{1}$. A simple calculation using Equation (2.1) and the fact that $G / K$ is fixed by $*$ verifies that

$$
x_{1}^{*} x_{1}+x_{3}^{*} x_{3}=A_{1}^{*}(X-\bar{\eta}) \bar{\alpha} \alpha^{*}(X-\eta) A_{1} .
$$

Taking determinants finishes the first part.

The second claim follows by observing both terms are complex numbers times their conjugates. The third is a special case of (1) since (2) allows square roots. For the fourth claim, set $x=g^{-1} \bar{n} l$. Using Equation (2.3) to calculate $g^{-1}$ and then expressing the result in the form $K(x) L(x) N(x)$, it is easy to see that if we write $K(x)=\left(\begin{array}{cc}a & b \\ -b & a\end{array}\right)$, then $(a+i b) L_{1}=$ $x_{1}-i x_{3}$ by looking at the upper left and lower left entries in the equality $x=K(x) L(x) N(x)$. A simple calculation then shows that

$$
(a+i b) L_{1}=-i \bar{\alpha}^{*}(X-\bar{\eta}) A_{1} .
$$

Taking determinants, noting that $\operatorname{det}(L)=|\operatorname{det}(L)|$, and using Equation (2.3) finishes the job.

We are now in a position to rewrite Theorem 3.1.

Definition 3.3. Let $f \in I_{p}^{+}$and $X \in H$. Define $\bar{f}$ a function on $H$ by the restriction to $\bar{N}$ :

$$
\bar{f}(X)=f\left(\left(\begin{array}{cc}
I & 0 \\
X & I
\end{array}\right)\right)
$$

Write $d X$ for Haar measure on $H$. 
Theorem 3.3. Let $f \in I_{p}^{+}, g \in G, X \in H, \eta=\eta_{g}$, and $\alpha=\alpha_{g}$. Then $S f \in C^{\infty}\left(G / K, \chi_{p}\right)$ can be calculated by

$$
S f(g)=i^{-n p} \operatorname{det}(\alpha)^{-p} \int_{H} \operatorname{det}(X-\eta)^{-p} \bar{f}(X) d X .
$$

Proof. From Theorem 3.1, Lemma 3.2 (with $l=I$ ), and Definitions 2.1 and 2.3 , it is easy to check that

$$
S f(g)=i^{-n p} \operatorname{det}\left(\bar{\alpha} \alpha^{*}\right)^{-p} \operatorname{det}\left(\bar{\alpha}^{*}\right)^{p} \int_{H} \operatorname{det}(X-\eta)^{-p} \bar{f}(X) d X .
$$

But Equation $(2.2)$ can be used to check that $\alpha \bar{\alpha}^{*}=\left(A A^{*}+B B^{*}\right)=\bar{\alpha} \alpha^{*}$ which finishes the proof.

\section{The Szegö Map to Functions on $\mathcal{D}^{+}$.}

Taking our cue from Lemma 2.2 and Theorem 3.3, it is reasonable to rewrite the Szegö map, $S$, and $C^{\infty}\left(G / K, \chi_{p}\right)$ in terms of the tube domain $\mathcal{D}^{+}$.

Definition 4.1. Let $g=\left(\begin{array}{cc}A & B \\ C & D\end{array}\right) \in G_{\mathbb{C}}$. Identify $H \cong \bar{N}$ and $\mathcal{D} \cong \bar{N}_{\mathbb{C}}$ by the map

$$
Z \rightarrow\left(\begin{array}{cc}
I & 0 \\
Z & I
\end{array}\right)
$$

This implements an embedding $\mathcal{D} \rightarrow G_{\mathbb{C}} / P_{\mathbb{C}}$ whose image is open and dense. For almost all $Z \in \mathcal{D}$, left multiplication by $g$ in $G_{\mathbb{C}} / P_{\mathbb{C}}$ may be pulled back to $\mathcal{D}$ by the linear fractional transformation action defined as

$$
g Z=(D Z+C)(B Z+A)^{-1} .
$$

Note that $H$ and $\mathcal{D}^{ \pm}$are $G$ orbits under this action and that $\eta_{g}$ from Lemma 2.2 is simply $g$ acting on $i I$.

If $\sigma \in C^{\infty}\left(G / K, \chi_{p}\right)$ does not vanish, there is an isomorphism

$$
C^{\infty}\left(G / K, \chi_{p}\right) \cong C^{\infty}(G / K)
$$

established by mapping $f \in C^{\infty}(G / K)$ to $\sigma f \in C^{\infty}\left(G / K, \chi_{p}\right)$. If we let

$$
\sigma(x, g)=\frac{\sigma\left(g^{-1} x\right)}{\sigma(x)}
$$

for $g \in G$ and $x \in G / K$ and define a $G$ action on $C^{\infty}(G / K)$ by

$$
g f(x)=\sigma(x, g) f\left(g^{-1} x\right),
$$

then the map $f \rightarrow f \sigma$ is a $G$ map as well. Below we choose a section $\sigma$ and use it to push the Szegö map down to functions on $G / K$. Finally, identify 
$G / K \cong \mathcal{D}^{+}$as in Lemma 2.2 by identifying $x \in G / K$ with $\eta=\eta_{x} \in \mathcal{D}^{+}$. Thus

$$
C^{\infty}(G / K) \cong C^{\infty}\left(\mathcal{D}^{+}\right) .
$$

Definition 4.2. Write $\eta=\eta_{g}$. Let $\sigma \in C^{\infty}\left(G / K, \chi_{p}\right)$ be defined by

$$
\sigma(g)=\mathrm{e}^{\left(\nu_{p}-\rho\right) H\left(g^{-1}\right)} \chi_{p}\left(K\left(g^{-1}\right)\right) \operatorname{det}(-\eta)^{p} .
$$

To make sure the above definition is valid, we check that

$$
\sigma(g k)=\chi_{p}(k)^{-1} \sigma(g)
$$

for $g \in G$ and $k \in K$. But this follows immediately by observing that $H\left(k^{-1} g^{-1}\right)=H\left(g^{-1}\right), K\left(k^{-1} g^{-1}\right)=k^{-1} K\left(g^{-1}\right)$ and that $\eta_{g k}=\eta_{g}$.

Lemma 4.1. For $g \in G$, write $\alpha=\alpha_{g}$. Then

$$
\sigma(g)=i^{-n p} \operatorname{det}(\alpha)^{-p} .
$$

Proof. This calculation follows from Lemma 3.2 with $\bar{n}=l=I$.

Since $C^{\infty}\left(G / K, \chi_{p}\right) \cong C^{\infty}\left(\mathcal{D}^{+}\right)$, we may view $C^{\infty}\left(\mathcal{D}^{+}\right)$as a $G$ space and view $S$ as the $G$-map taking $I_{p}^{+} \rightarrow C^{\infty}\left(\mathcal{D}^{+}\right)$. We continue to denote the resulting map as $S$ as the range will remove ambiguity. We now apply Equations (4.1) and (4.4) and Lemma 4.1 to rewrite Theorem 3.3 in terms of $C^{\infty}\left(\mathcal{D}^{+}\right)$.

Theorem 4.2. The $G$ intertwining map $S: I_{p}^{+} \rightarrow C^{\infty}\left(\mathcal{D}^{+}\right)$acts by

$$
S f(\eta)=\int_{H} \operatorname{det}(X-\eta)^{-p} \bar{f}(X) d X .
$$

It is useful to write the $G$ action on $C^{\infty}\left(\mathcal{D}^{+}\right)$explicitly.

Lemma 4.3. Identifying $G / K \cong \mathcal{D}^{+}$and writing $g=\left(\begin{array}{cc}A & B \\ C & D\end{array}\right) \in G$,

$$
\sigma(\eta, g)=\operatorname{det}\left(D^{*}-B^{*} \eta\right)^{-p} .
$$

Proof. It is enough to compute $\sigma(\cdot, g)$ on $\bar{N} L$. Writing

$$
x=\left(\begin{array}{cc}
I & 0 \\
X & I
\end{array}\right)\left(\begin{array}{cc}
L & 0 \\
0 & L^{*-1}
\end{array}\right)
$$

it is easy to compute

$$
\alpha\left(g^{-1} x\right) \alpha(x)^{-1}=D^{*}-B^{*} \eta_{x} .
$$

Applying Lemma 4.1 to Equation (4.2) finishes the proof.

Thus using Lemma 3.2 and Equation (4.3), we can write the $G$ action on $C^{\infty}\left(\mathcal{D}^{+}\right)$. 
Theorem 4.4. For $f \in C^{\infty}\left(\mathcal{D}^{+}\right), \eta \in \mathcal{D}^{+}$, and $g=\left(\begin{array}{cc}A & B \\ C & D\end{array}\right) \in G$,

$$
g f(\eta)=\operatorname{det}\left(D^{*}-B^{*} \eta\right)^{-p} f\left(g^{-1} \eta\right) .
$$

5. $I_{p}^{ \pm}$as Functions on $H$.

This section looks at an explicit form of the closure of $I_{p}^{ \pm}$.

Lemma 5.1. Restriction to $\bar{N} \cong H$ establishes an isomorphism of $G$ spaces between the closure of

$$
I_{p}^{ \pm}
$$

and

$$
L^{2}\left(H, \operatorname{det}\left(I+X^{2}\right)^{ \pm(n-p)} d X\right)
$$

where $d X$ is Haar measure on $H$.

Proof. Apply Lemma 3.2 (with $g=l=I$ ) to Equation (2.6) and make the identification of $H$ with $\bar{N}$ as in Definition 4.1.

In this section we explicitly compute the action of $G$ on $L^{2}(H, \operatorname{det}(I+$ $\left.\left.X^{2}\right)^{ \pm(n-p)} d X\right)$ and extend the Szegö map accordingly.

Definition 5.1. Write

$$
L^{2}(H)^{ \pm}=L^{2}\left(H, \operatorname{det}\left(I+X^{2}\right)^{ \pm(n-p)} d X\right)
$$

and

$$
L^{2}(H)=L^{2}(H, d X) .
$$

Lemma 5.2. $L^{2}(H)^{+} \subseteq L^{2}(H) \subseteq L^{2}(H)^{-}$.

Proof. Since $0 \leq p \leq n$ and $\operatorname{det}\left(I+X^{2}\right) \geq 1$,

$$
0<\operatorname{det}\left(I+X^{2}\right)^{-(n-p)} \leq 1 \leq \operatorname{det}\left(I+X^{2}\right)^{+(n-p)} .
$$

Thus the Lemma follows immediately from the definition of $L^{2}(H)^{+}$in Definition 5.1.

For functions $f \in I_{p}^{ \pm}$, this Lemma can also be proved directly. Since two formulas arising from this approach will be needed later, we sketch the idea. For instance, by starting with $f \in I_{p}^{+}$, applying Equation (2.5) to the $K M A N$ decomposition, and using Lemma 3.2 (with $g=l=I$ ) it is easy to show

$$
|\bar{f}(X)|^{2} \leq C \operatorname{det}\left(I+X^{2}\right)^{p-2 n}
$$


where $C$ is a constant bounding $|f|^{2}$ on $K$. This is enough to finish the first inclusion since it is known $([7], \S 2.1$, p. 38) that

$$
\int_{H} \operatorname{det}\left(I+X^{2}\right)^{m} d X<\infty
$$

whenever $m<-n+\frac{1}{2}$. Though not needed immediately, we will also have recourse to make use of a formula for the Jacobian of the change of variables on $H$ given by $X \rightarrow g X$. It is

$$
\operatorname{det}(B X+A)^{-2 n} .
$$

It is now apparent that Theorem 4.2 may be completed to the following (remember everything can be written as an integral over $K$ ).

Theorem 5.3. The $G$ intertwining map $S: L^{2}(H)^{+} \rightarrow C^{\infty}\left(\mathcal{D}^{+}\right)$acts by

$$
S f(\eta)=\int_{H} \operatorname{det}(X-\eta)^{-p} f(X) d X .
$$

We finish this section by writing the $G$ action on $L^{2}(H)^{ \pm}$explicitly.

Lemma 5.4. If $g=\left(\begin{array}{cc}A & B \\ C & D\end{array}\right) \in G$ and $X \in H$, then $\operatorname{det}(A+B X)$, $\operatorname{det}(D-X B) \in \mathbb{R}$.

Proof. Recall $g X \in H$ by Definition 4.1. Thus

$$
g^{\prime}=\left(\begin{array}{cc}
I & 0 \\
-g X & I
\end{array}\right) g\left(\begin{array}{cc}
I & 0 \\
X & I
\end{array}\right) \in G .
$$

In fact it is easy to calculate that $g^{\prime}=\left(\begin{array}{cc}A+B X & * \\ 0 & -(g X) B+D\end{array}\right)$ and so $g^{\prime} \in P$. Equation (2.4) finishes the theorem. As an additional point we see that

$$
(A+B X)^{-1}=D^{*}-B^{*}(g X)
$$

whenever it is invertible. To prove the second assertion, apply the first to $g^{-1}$ and then apply $(\cdot)^{*}$.

Theorem 5.5. Let $g=\left(\begin{array}{cc}A & B \\ C & D\end{array}\right) \in G$. If $f_{ \pm} \in L^{2}(H)^{ \pm}$, then

$$
g f_{+}(X)=\operatorname{det}(D-X B)^{-2 n+p} f_{+}\left(g^{-1} X\right)
$$

and

$$
g f_{-}(X)=\operatorname{det}(D-X B)^{-p} f_{-}\left(g^{-1} X\right)
$$


Proof. If we write $g^{-1}\left(\begin{array}{cc}I & 0 \\ X & I\end{array}\right)$ in the form

$$
\left(\begin{array}{cc}
I & 0 \\
Y & I
\end{array}\right)\left(\begin{array}{cc}
L & 0 \\
0 & L^{*-1}
\end{array}\right)\left(\begin{array}{cc}
I & Y^{\prime} \\
0 & I
\end{array}\right)
$$

we can solve

$$
\begin{gathered}
Y=g^{-1} X, \\
L=D^{*}-B^{*} X,
\end{gathered}
$$

and $Y^{\prime}=\left(-C^{*}+A^{*} X\right)^{-1}\left(I-L^{*-1}\right)$. For $f \in I_{p}^{+}$, this says that $g f(X)=$ $\operatorname{sgn}\left(\operatorname{det}\left(D^{*}-B^{*} X\right)\right)^{-p}\left|\operatorname{det}\left(D^{*}-B^{*} X\right)\right|^{-2 n+p} f\left(g^{-1} X\right)$. Equation (2.4) applied to $g^{-1}$ shows that $\operatorname{det}\left(D^{*}-B^{*} X\right) \in \mathbb{R}$. We can therefore change the $-p$ in the previous formula to $-2 n+p$ since the parity does not change modulo 2. Hence we get that $g f(X)=\operatorname{det}\left(D^{*}-B^{*} X\right)^{-2 n+p} f\left(g^{-1} X\right)$ which is equal to $\operatorname{det}(D-X B)^{-2 n+p} f\left(g^{-1} X\right)$. The work for $I_{p}^{-}$is done similarly by replacing the $-2 n+p$ by $-p$.

\section{The Orbits $\mathcal{O}_{p}$.}

Let

$$
l=\left(\begin{array}{cc}
A & 0 \\
0 & A^{*-1}
\end{array}\right) \in L
$$

When convenient, we make use of the identification

$$
L \cong\left\{A \in G l(n, \mathbb{C}) \mid \operatorname{det}(A) \in \mathbb{R}^{\times}\right\}
$$

implemented by $l \rightarrow A$ above. Definition 4.1 calculates the action of $L$ on $\bar{N}$ which pulls down to an action of $L$ on $H$ as

$$
l \cdot X=A^{-1^{*}} X A^{-1}
$$

for $X \in H$. Hence the $L$-orbits on $H$ are parameterized by signature. The study of these orbits will be of fundamental importance.

Definition 6.1. Let $\mathcal{O}_{p}$ be the $L$-orbit in $H$ consisting of Hermitian matrices of signature $p, 0$.

For general reasons, there exist $L$-equivariant measures on $\mathcal{O}_{p}([\mathbf{1 3}])$, but they are also easy to write explicitly. We briefly outline their construction. In this paragraph only, write $\mathcal{O}_{p}(n)$ for the matrices in $H(n)$ of signature $p, 0$. Then there is a smooth emedding $\mathbb{C}^{p(n-p)} \times \mathcal{O}_{p}(p) \rightarrow \mathcal{O}_{p}(n)$ with dense open image given by

$$
(Z, X) \rightarrow\left(\begin{array}{ll}
I_{p} & 0 \\
Z^{*} & I_{n-p}
\end{array}\right)\left(\begin{array}{ll}
X & 0 \\
0 & 0
\end{array}\right)\left(\begin{array}{ll}
I_{p} & Z \\
0 & I_{n-p}
\end{array}\right) .
$$


Using this embedding, the desired $L$-equivariant measure on $\mathcal{O}_{p}$ (up to a scalar multiple) pulls back to

$$
\operatorname{det}(X)^{n-p} d Z_{p} d X_{p}
$$

where $d Z_{p}$ is Haar measure on $\mathbb{C}^{p(n-p)}$ and $d X_{p}$ is Haar measure on $H(p)$ restricted to the open orbit $\mathcal{O}_{p}(p)$. We omit the details.

Definition 6.2. Write $\tau$ for the character on $L$ acting by $\tau(l)=\operatorname{det}(A)$.

1) Let

$$
d \mu_{p}
$$

be the unique (up to scalar multiplication) $L$-equivariant measure on $\mathcal{O}_{p}$ that transforms by the character $\tau^{2 p}$ (so the change of variables $l \cdot X \rightarrow X$ multiplies the measure by $\left.\operatorname{det}(A)^{2 p}\right)$. The measure $d \mu_{p}$ will be normalized below.

2) Write

$$
L^{2}\left(\mathcal{O}_{p}\right)=L^{2}\left(\mathcal{O}_{p}, d \mu_{p}\right)
$$

Normalize $d \mu_{p}$ so that $\int_{\mathcal{O}_{p}} \mathrm{e}^{-\operatorname{tr}(Y)} d \mu_{p}(Y)=1$. This allows us to verify the following well known identity.

Lemma 6.1. Let $Z \in \mathcal{D}^{+}$. Then

$$
i^{n p} \operatorname{det}(Z)^{-p}=\int_{\mathcal{O}_{p}} \mathrm{e}^{i \operatorname{tr}(Z \xi)} d \mu_{p}(\xi) .
$$

Proof. We first show

$$
\operatorname{det}(Y)^{-p}=\int_{\mathcal{O}_{p}} \mathrm{e}^{\operatorname{tr}(Y \xi)} d \mu_{p, 0}(\xi)
$$

for any $Y \in H^{+}$. Using the $L$ action from (6.2), write $Y=A^{-1 *} A^{-1}$ with $\operatorname{det}(A) \in \mathbb{R}^{\times}$. Then, making use of the $L$-equivariance and normalization, we calculate:

$$
\begin{aligned}
\int_{\mathcal{O}_{p}} \mathrm{e}^{-\operatorname{tr}(Y \xi)} d \mu_{p}(\xi) & =\int_{\mathcal{O}_{p}} \mathrm{e}^{-\operatorname{tr}\left(A^{-1 *} A^{-1} \xi\right)} d \mu_{p}(\xi) \\
& =\int_{\mathcal{O}_{p}} \mathrm{e}^{-\operatorname{tr}\left(A^{-1} \xi A^{-1 *}\right)} d \mu_{p}(\xi) \\
& =\operatorname{det}\left(A^{*}\right)^{2 p} \int_{\mathcal{O}_{p}} \mathrm{e}^{-\operatorname{tr}(\xi)} d \mu_{p}(\xi) \\
& =\operatorname{det}(Y)^{-p}
\end{aligned}
$$

To finish the Lemma, write $Z=X+i Y$ with $X \in H$ and $Y \in H^{+}$. We see $-i Z=Y-i X$. The statement of the Lemma then follows by analytic continuation. 
To apply this Lemma in the setting of our Szegö map, we need the Fourier transform.

Definition 6.3. Write $\mathcal{S}(H)$ for the set of all $S$ chwartz functions on $H$. As this space is not $G$ invariant, write $\mathcal{S}(H)^{+}$for the smallest $G$ invariant space containing $\mathcal{S}(H)$ :

$$
\mathcal{S}(H)^{+}=\operatorname{span}\left\{g \phi \mid g \in G, \phi \in \mathcal{S}(H) \subseteq L^{2}(H)^{+}\right\} .
$$

For $\phi \in \mathcal{S}(H)$, define its Fourier transform, $\widehat{\phi} \in \mathcal{S}(H)$, by

$$
\widehat{\phi}(\xi)=\int_{H} \mathrm{e}^{i \operatorname{tr}(\xi X)} \phi(X) d X
$$

and, up to a scalar multiple, the inverse Fourier transform, $\check{\phi} \in \mathcal{S}(H)$, by

$$
\check{\phi}(\xi)=\int_{H} \mathrm{e}^{-i \operatorname{tr}(\xi X)} \phi(X) d X .
$$

Since we will eventually be looking at boundary values of the map $S$, the following Lemma will be needed.

Lemma 6.2. Let $\phi \in \mathcal{S}(H), Z \in \mathcal{D}^{+}$, and $Y \in H$. Then

$$
\lim _{Z \rightarrow Y} \int_{H} \operatorname{det}(X+Z)^{-p} \phi(X) d X=i^{-n p} \int_{\mathcal{O}_{p}} \mathrm{e}^{i \operatorname{tr}(Y \xi)} \widehat{\phi}(\xi) d \xi .
$$

Proof. We begin by using Lemma 6.1 and compute

$$
\int_{H} \operatorname{det}(X+Z)^{-p} \phi(X) d X=i^{-n p} \int_{H} \int_{\mathcal{O}_{p}} \mathrm{e}^{i \operatorname{tr}(X+Z) \xi} \phi(X) d \mu_{p}(\xi) d X .
$$

To apply Fubini's theorem, we need to check the $L^{1}$ condition. Write $Z=X^{\prime}+i Y$ with $X^{\prime} \in H$ and $Y \in H^{+}$and use Lemma 6.1:

$$
\begin{aligned}
\int_{H} \int_{\mathcal{O}_{p}}\left|\mathrm{e}^{i \operatorname{tr}(X+Z) \xi} \phi(X)\right| d \mu_{p}(\xi) d X & =\int_{H} \int_{\mathcal{O}_{p}} \mathrm{e}^{-\operatorname{tr}(Y \xi)}|\phi(X)| d \mu_{p}(\xi) d X \\
& =\operatorname{det}(Y)^{-p} \int_{\mathcal{O}_{p}}|\phi(X)| d X<\infty .
\end{aligned}
$$

Hence

$$
\begin{aligned}
\int_{H} \int_{\mathcal{O}_{p}} \mathrm{e}^{i \operatorname{tr}(X+Z) \xi} \phi(X) d \mu_{p}(\xi) d X & =\int_{\mathcal{O}_{p}} \mathrm{e}^{i \operatorname{tr}(Z \xi)} \int_{H} \mathrm{e}^{i \operatorname{tr}(X \xi)} \phi(X) d X d \mu_{p}(\xi) \\
& =\int_{\mathcal{O}_{p}} \mathrm{e}^{i \operatorname{tr}(Z \xi)} \widehat{\phi}(\xi) d \mu_{p}(\xi) .
\end{aligned}
$$

Since $\widehat{\phi}$ is still Schwartz and the measure (see Equation (6.3)) is only of polynomial growth, the above integrand is an $L^{1}$ function. Hence, when we take the limit as $Z \rightarrow Y$, we may move the limit past the integral to finish the Lemma. 


\section{Boundary Values of the Szegö Map.}

Definition 7.1. For $f \in C^{\infty}\left(\mathcal{D}^{+}\right)$and $X \in H$, define

$$
B f(X)=\lim _{Z \rightarrow X} f(Z)
$$

where $Z \in \mathcal{D}^{+}$.

In general, $B f$ may not be well defined. However, we see below that it is at least well behaved on $\operatorname{Im}(S)$.

Theorem 7.1. Let $\phi \in \mathcal{S}(H)$ and $\eta \in \mathcal{D}^{+}$. Then

$$
S \phi(\eta)=i^{n p} \int_{\mathcal{O}_{p}} \mathrm{e}^{i \operatorname{tr} \eta \xi} \check{\phi}(\xi) d \xi .
$$

Moreover, $B$ is well defined on $S(\mathcal{S}(H))$ and $B S \phi$ is alternately written as the smooth function

$$
B S \phi(Y)=i^{n p} \int_{\mathcal{O}_{p}} \mathrm{e}^{i \operatorname{tr}(Y \xi)} \check{\phi}(\xi) d \xi .
$$

Proof. Lemma 6.2 computes that

$$
\begin{aligned}
& \lim _{Z \rightarrow Y} \int_{H} \operatorname{det}(X+Z)^{-p} \phi(X) d X \\
& =i^{-n p} \lim _{Z \rightarrow Y} \int_{\mathcal{O}_{p}} \mathrm{e}^{i \operatorname{tr} Z W} \widehat{\phi}(W) d \mu_{p}(W) \\
& =i^{-n p} \int_{\mathcal{O}_{p}} \mathrm{e}^{i \operatorname{tr}(Y W)} \widehat{\phi}(W) d \mu_{p}(W) .
\end{aligned}
$$

Multiplying both sides by $(-1)^{n p}$ and making the change of variables $X \rightarrow$ $-X$ finishes the identity. Regarding smoothness, recall that $\widehat{\phi}$ is still Schwartz and the measure (see Equation (6.3)) is only of polynomial growth so that the integrand is an $L^{1}$ function.

Definition 7.2. If $f \in \mathcal{S}(H)^{+}$, define $A f$ by

$$
A f=B S f \text {. }
$$

So far this map is well defined on $\mathcal{S}(H)$ by Theorem 7.1.

Theorem 7.2. Let $f \in \mathcal{S}(H)^{+}$and $\psi=S f$. Then (1) Af and $B \psi$ are well defined almost everywhere; (2) $A f=B \psi \in L^{2}(H)^{-}$; and (3) $A$ and $B$ are $G$-maps on $\mathcal{S}(H)^{+}$and $S\left(\mathcal{S}(H)^{+}\right)$, respectively. Finally, for $\phi \in \mathcal{S}(H)$, $\eta \in \mathcal{D}$, and $Y \in H$,

$$
S \phi(\eta)=i^{n p} \int_{\mathcal{O}_{p}} \mathrm{e}^{i \operatorname{tr} \eta \xi} \check{\phi}(\xi) d \xi
$$

and

$$
A \phi(Y)=i^{n p} \int_{\mathcal{O}_{p}} \mathrm{e}^{i \operatorname{tr}(Y \xi)} \check{\phi}(\xi) d \xi
$$


Proof. Begin with any function $\psi \in C^{\infty}\left(\mathcal{D}^{+}\right)$for which $B \psi$ is well defined. First we check $B$ commutes with the group actions given in Theorems 5.5 and 4.4. Write $g=\left(\begin{array}{cc}A & B \\ C & D\end{array}\right) \in G$. Then using Theorems 5.5 and 4.4 for the group action and making use of Lemma 5.4 in the second set of equations below, we calculate:

$$
\begin{aligned}
g B \psi(X) & =\operatorname{det}(D-X B)^{-p} B \psi\left(g^{-1} X\right) \\
& =\operatorname{det}(D-X B)^{-p} \lim _{Z \rightarrow g^{-1} X} \psi(Z) .
\end{aligned}
$$

On the other hand, we have

$$
\begin{aligned}
B g \psi(X) & =\lim _{\zeta \rightarrow X} g \psi(\zeta) \\
& =\lim _{\zeta \rightarrow X} \operatorname{det}\left(D^{*}-B^{*} \zeta\right)^{-p} \psi\left(g^{-1} \zeta\right) \\
& =\overline{\operatorname{det}(D-X B)}-p \lim _{\zeta \rightarrow X} \psi\left(g^{-1} \zeta\right) \\
& =\operatorname{det}(D-X B)^{-p} \lim _{Z \rightarrow g^{-1} X} \psi(Z)
\end{aligned}
$$

so that $g B \psi=B g \psi$ which proves part (3) since $S$ is a $G$-map (Theorem 5.3). Coupled with Theorem 7.1, Equations (7.2) show $B g \psi$ is well defined almost everywhere. Since $S$ is a $G$-map, this finishes part (1). That the range of $B$ restricted to $\mathcal{S}(H)^{+}$is contained in $L^{2}(H)^{-}$follows from the $G$ action. The argument is completely analogous to the one around Equation (5.1) (since each $f \in \mathcal{S}(H)^{+}$comes from the smooth principal series) except that the final bound will be

$$
|B f(X)|^{2} \leq C \operatorname{det}\left(I+X^{2}\right)^{-p}
$$

The final equations come from Theorem 7.1.

Note that though $B f$ is well defined almost everywhere for $\psi \in S\left(\mathcal{S}(H)^{+}\right)$, it need not be given by the formula in Theorem 7.1 for $\psi \notin S(\mathcal{S}(H))$. A similar cautionary remark applies to $A$ on $\mathcal{S}(H)$ versus $\mathcal{S}(H)^{+}$.

Theorem 7.2 establishes the following commutative diagram of $G$ maps:

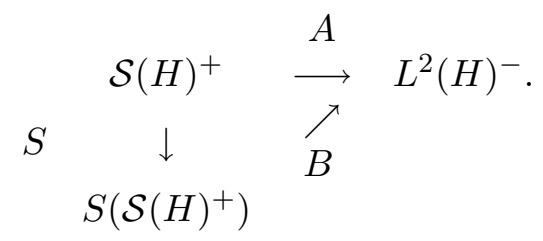




\section{Functions on $\mathcal{O}_{p}$ and an Inner Product.}

Definition 8.1. For $\phi \in \mathcal{S}(H)$, define $F_{R} \phi \in L^{2}\left(\mathcal{O}_{p}, d \mu_{p}\right)$ ( $F$ for Fourier transform and $R$ for restriction) by

$$
F_{R} \phi\left(\xi_{p}\right)=\check{\phi}\left(\xi_{p}\right) .
$$

For $\psi \in \operatorname{Im}\left(F_{R}\right)$, define $F_{E} \psi \in L^{2}(H)^{-}$(E for extension $)$by

$$
F_{E} \psi(X)=i^{n p} \int_{\mathcal{O}_{p}} \mathrm{e}^{i \operatorname{tr}(\xi X)} \psi(\xi) d \mu_{p}(\xi) .
$$

First note that $\widehat{\phi}$ is still Schwartz and the measure $d \mu_{p}$ (see Equation (6.3)) is only of polynomial growth so that $F_{E} \psi$ is well defined. Second, note that Theorem 7.2 immediately implies that on $\mathcal{S}(H)$,

$$
F_{E} \circ F_{R}=\left.A\right|_{\mathcal{S}(H)}=\left.B \circ S\right|_{\mathcal{S}(H)}
$$

where $\left.A\right|_{\mathcal{S}(H)}$ denotes the map $A$ restricted to $\mathcal{S}(H)$. In other words, there is a commutative diagram of maps (compare to diagram 7.4)

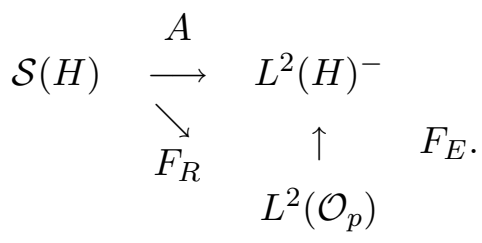

Also note that $L^{2}\left(\mathcal{O}_{p}\right)$ comes equipped with its own inner product denoted by

$$
\langle\cdot, \cdot\rangle_{\mathcal{O}_{p}}
$$

This pairing can be related to $A$ as follows.

Definition 8.2. If $\phi_{1}, \phi_{2} \in \mathcal{S}(H)^{+}$, let

$$
\left\langle\phi_{1}, \phi_{2}\right\rangle_{A}=i^{n p} \int_{H} \phi_{1}(X) \overline{A \phi_{2}(X)} d X .
$$

Equations (5.1), (7.3), and (5.2) can be used to show that $\langle\cdot, \cdot\rangle_{A}$ is well defined for functions coming from the principal series $I_{p}^{+}$which includes $\mathcal{S}(H)^{+}$ (see the proof of Lemma 10.1 or a more general result under Definition 10.2 below).

Theorem 8.1. If $\phi_{1}, \phi_{2} \in \mathcal{S}(H)$, then

$$
\left\langle\phi_{1}, \phi_{2}\right\rangle_{A}=\left\langle F_{R} \phi_{1}, F_{R} \phi_{2}\right\rangle_{\mathcal{O}_{p}} .
$$

Moreover, the form $\langle\cdot, \cdot\rangle_{A}$ is $G$-invariant on $\mathcal{S}(H)^{+}$. 
Proof. We make use of Theorem 7.2 to calculate:

$$
\begin{aligned}
\left\langle\phi_{1}, \phi_{2}\right\rangle_{A} & =i^{n p} \int_{H} \phi_{1}(X) \overline{A \phi_{2}(X)} d X \\
& =\int_{H} \int_{\mathcal{O}_{p}} \phi_{1}(X) \mathrm{e}^{-i \operatorname{tr}(X \xi)} \overline{\check{\phi}(\xi)} d \xi d X \\
& =\int_{\mathcal{O}_{p}} \int_{H} \phi_{1}(X) \mathrm{e}^{-i \operatorname{tr}(X \xi)} \overline{\grave{\phi}(\xi)} d \xi d X \\
& =\int_{\mathcal{O}_{p}} \check{\phi}_{1}(\xi) \overline{\check{\phi}_{2}(\xi)} d \mu_{p}(\xi) .
\end{aligned}
$$

To check $G$-invariance, write $g=\left(\begin{array}{cc}A & B \\ C & D\end{array}\right) \in G$. In the following equations, make use of the actions given in Theorem 5.5, Equation (5.3) for the change of variables, Lemma 5.4 for conjugation issues, and Equation (2.1) and Definition 4.1 to check that $\left(D^{*}-B^{*} g X\right)=(B X+A)^{-1}$ :

$$
\begin{aligned}
\left\langle g \phi_{1}, g \phi_{2}\right\rangle & =i^{n p} \int_{H} g \phi_{1}(X) \overline{A g \phi_{2}(X)} d X \\
& =i^{n p} \int_{H} \operatorname{det}(D-X B)^{-2 n+p} \phi_{1}\left(g^{-1} X\right) \overline{g A \phi_{2}(X)} d X \\
& =i^{n p} \int_{H} \operatorname{det}(D-X B)^{-2 n} \phi_{1}\left(g^{-1} X\right) \overline{A \phi_{2}\left(g^{-1} X\right)} d X \\
& =i^{n p} \int_{H} \operatorname{det}\left(D^{*}-B^{*} X\right)^{-2 n} \phi_{1}\left(g^{-1} X\right) \overline{A \phi_{2}\left(g^{-1} X\right)} d X \\
& =i^{n p} \int_{H} \operatorname{det}\left(D^{*}-B^{*} g X\right)^{-2 n} \operatorname{det}(B X+A)^{-2 n} \phi_{1}(X) \overline{A \phi_{2}(X)} d X \\
& =i^{n p} \int_{H} \phi_{1}(X) \overline{A \phi_{2}(X)} d X \\
& =\left\langle\phi_{1}, \phi_{2}\right\rangle .
\end{aligned}
$$

The equality of the two pairings in the above Theorem will be extended to a larger domain as soon as the map $F_{R}$ is extended.

\section{Continuity of $A, F_{R}$, and $F_{E}$.}

As it stands, most operators are only defined on dense sets such as $\mathcal{S}(H) \subseteq$ $L^{2}(H)^{+}$. To complete the picture, we need to prove the operators are continuous.

Theorem 9.1. The maps

$$
A: \mathcal{S}(H) \subseteq L^{2}(H)^{+} \rightarrow L^{2}(H)^{-},
$$




$$
F_{R}: \mathcal{S}(H) \subseteq L^{2}(H)^{+} \rightarrow L^{2}\left(\mathcal{O}_{p}\right)
$$

and

$$
F_{E}: \operatorname{Im}\left(\left.F_{R}\right|_{\mathcal{S}(H)}\right) \subseteq L^{2}\left(\mathcal{O}_{p}\right) \rightarrow L^{2}(H)^{-}
$$

are continuous maps. The notation $\operatorname{Im}\left(\left.F_{R}\right|_{\mathcal{S}(H)}\right)$ denotes the image of $F_{R}$ restricted to $\mathcal{S}(H)$.

This section is devoted to the proof of Theorem 9.1. The first step is the following Lemma.

Lemma 9.2. If the operator

$$
F_{E}: \operatorname{Im}\left(\left.F_{R}\right|_{\mathcal{S}(H)}\right) \subset L^{2}\left(\mathcal{O}_{p}\right) \rightarrow L^{2}(H)^{-}
$$

is continuous, then

$$
A: \mathcal{S}(H) \subseteq L^{2}(H)^{+} \rightarrow L^{2}(H)^{-}
$$

and

$$
F_{R}: \mathcal{S}(H) \subseteq L^{2}(H)^{+} \rightarrow L^{2}\left(\mathcal{O}_{p}\right)
$$

are bounded operators as well.

Proof. Suppose the hypothesis of this Lemma is in effect and let $f \in \mathcal{S}(H)$. Consider the map $A$ first. Then for some constant $C$,

$$
\|A f\|_{L^{2}(H)^{-}}^{2}=\left\|F_{E} F_{R} f\right\|_{L^{2}(H)^{-}}^{2} \leq C\left\|F_{R} f\right\|_{L^{2}\left(\mathcal{O}_{p}\right)^{2}}^{2} .
$$

On the other hand, $\left\|F_{R} f\right\|_{L^{2}\left(\mathcal{O}_{p}\right)}^{2}=\left\langle F_{R} f, F_{R} f\right\rangle_{\mathcal{O}_{p}}=\langle f, f\rangle_{A}$ by Theorem 8.1. But Definition 8.2 says

$$
\begin{aligned}
\langle f, f\rangle_{A} & =\left|\langle f, f\rangle_{A}\right|=\left|\int_{H} f(X) \overline{A f(X)} d X\right| \\
& \leq \int_{H}\left|\left[f(X) \operatorname{det}\left(I+X^{2}\right)^{\frac{n-p}{2}}\right]\left[\overline{A f(X)} \operatorname{det}\left(I+X^{2}\right)^{\frac{-n+p}{2}}\right]\right| d X \\
& \leq\|f\|_{L^{2}(H)^{+}}\|A f\|_{L^{2}(H)^{-}} .
\end{aligned}
$$

Putting these equations together gives

$$
\|A f\|_{L^{2}(H)^{-}}^{2} \leq C\|f\|_{L^{2}(H)^{+}}\|A f\|_{L^{2}(H)^{-}} .
$$

Division finishes the proof for the continuity of $A$.

Now consider $F_{R}$. Using the relations above, $\left\|F_{R} f\right\|_{L^{2}\left(\mathcal{O}_{p}\right)}^{2}=\langle f, f\rangle_{A} \leq$ $\|f\|_{L^{2}(H)^{+}}\|A f\|_{L^{2}(H)^{-}} \leq C\|f\|_{L^{2}(H)^{+}}^{2}$. Division again finishes the proof for continuity of $F_{R}$.

Thus we devote the rest of the section to proving that $F_{E}: \operatorname{Im}\left(\left.F_{R}\right|_{\mathcal{S}(H)}\right) \subset$ $L^{2}\left(\mathcal{O}_{p}\right) \rightarrow L^{2}(H)^{-}$is a bounded map. This amounts to showing that

$$
\int_{H}|A f(X)|^{2} \operatorname{det}\left(I+X^{2}\right)^{-n+p} d X \leq C\|\check{f}\|_{L^{2}\left(\mathcal{O}_{p}\right)}
$$


for some constant, $C$, and all $f \in \mathcal{S}(H)$. In the special case of $p=n$, this statement is trivial to verify using the Plancherel theorem as $\mathcal{O}_{n}$ is open in $H, d \mu_{n}=\left.d X\right|_{\mathcal{O}_{n}}$, and $\operatorname{det}\left(I+X^{2}\right)^{ \pm(n-p)}=1$. In the case of $p<n$, much more work is required.

Let $S_{p}$ be the stabilizer of

$$
E_{p}=\left(\begin{array}{cc}
I_{p} & \\
& 0_{n-p}
\end{array}\right)
$$

in $L$ so that $\mathcal{O}_{p} \cong L / S_{p}$ and let $\widetilde{S}_{n-p}$ be the stabilizer of

$$
\widetilde{E}_{n-p}=\left(\begin{array}{cc}
0 p & \\
& I_{n-p}
\end{array}\right)
$$

so that $\mathcal{O}_{n-p} \cong L / \widetilde{S}_{n-p}$.

Lemma 9.3. Given a smooth function of compact support, $f$, on $\mathcal{O}_{p} \times \mathcal{O}_{n-p}$, pull and push it to a function $f^{*}$ on $\mathcal{O}_{n}$ using the double fibration:

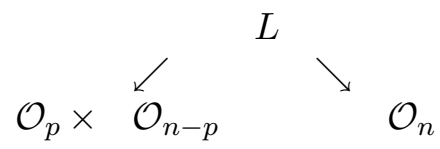

by

$$
f^{*}\left(l \cdot E_{n}\right)=\int_{S_{n} / S_{p} \cap \widetilde{S}_{n-p}} f\left((l s) \cdot E_{p},(l s) \cdot \widetilde{E}_{n-p}\right) d s
$$

for $l \in L$ where $d s$ is an $S_{n}$-invariant measure. The function $f^{*}$ satisfies

$$
\int_{\mathcal{O}_{n}} f^{*}\left(\xi_{n}\right) d \mu_{n}\left(\xi_{n}\right)=\int_{\mathcal{O}_{p} \times \mathcal{O}_{n-p}} f\left(\xi_{p}, \xi_{n-p}\right) d \mu_{p}\left(\xi_{p}\right) d \mu_{n-p}\left(\xi_{n-p}\right) .
$$

In particular, if $f_{p}$ and $f_{n-p}$ are functions on $\mathcal{O}_{p}$ and $\mathcal{O}_{n-p}$, respectively, then $f_{p} \times f_{n-p}$ is a function on $\mathcal{O}_{p} \times \mathcal{O}_{n-p}$. Define

$$
f_{p} * f_{n-p}
$$

to be the function on $\mathcal{O}_{n}$ given by $\left(f_{p} \times f_{n-p}\right)^{*}$.

Proof. Easy calculations show that $S_{n} / S_{p} \cap \widetilde{S}_{n-p} \cong U(n) / U(p) \times U(n-p)$ so that $d s$ exists since have a quotient of reductive groups. Then it is easy to see that it suffices to prove the injection

$$
L / S_{p} \cap \widetilde{S}_{n-p} \rightarrow \mathcal{O}_{p} \times \mathcal{O}_{n-p}
$$

induced by the diagonal action, $l \rightarrow\left(l \cdot E_{p}, l \cdot \widetilde{E}_{n-p}\right)$, has a dense open image. Using the transitivity of the $L$-action on $\mathcal{O}_{p}$, it suffices to show that $S_{p}$ can be used to conjugate almost all elements in $\mathcal{O}_{n-p}$ to $\widetilde{E}_{n-p}$. But for this, it suffices to show that almost all $A \in G l(n, \mathbb{C})$, with $\operatorname{det}(A) \in \mathbb{R}^{\times}$, can be written as the product of elements from $S_{p}$ and $\widetilde{S}_{n-p}$. But this is an easy calculation we omit. 
By previous remarks, the following Lemma will finish the proof of Theorem 9.1.

Theorem 9.4. For some constant, $C$, and all $f \in \mathcal{S}(H)$,

$$
\int_{H}|A f(X)|^{2} \operatorname{det}\left(I+X^{2}\right)^{-n+p} d X \leq C\|\check{f}\|_{L^{2}\left(\mathcal{O}_{p}\right)} .
$$

Proof. By Theorem 7.2, we know $\int_{H}|A f(X)|^{2} \operatorname{det}\left(I+X^{2}\right)^{-n+p} d X$ is equal to

$$
\int_{H}\left|\int_{\mathcal{O}_{p}} \mathrm{e}^{i \operatorname{tr}(X \xi)} \check{f}(\xi) d \mu_{p}(\xi)\right|^{2} \operatorname{det}\left(I+X^{2}\right)^{-n+p} d X .
$$

On the other hand, Lemma 6.1 shows that

$$
|\operatorname{det}(i I+X)|^{-n+p}=\left|\int_{\mathcal{O}_{n-p}} \mathrm{e}^{i \operatorname{tr}[(i I+X) \xi]} d \mu_{n-p}(\xi)\right| .
$$

Since $\operatorname{det}\left(I+X^{2}\right)=\operatorname{det}(i I+X) \operatorname{det}(-i I+X)$ and $|\operatorname{det}(i I+X)|=$ $|\operatorname{det}(-i I+X)|$, we therefore know

$$
\operatorname{det}\left(I+X^{2}\right)^{-n+p}=\left|\int_{\mathcal{O}_{n-p}} \mathrm{e}^{i \operatorname{tr}[(i I+X) \xi]} d \mu_{n-p}(\xi)\right|^{2} .
$$

Thus $\int_{H}|A f(X)|^{2} \operatorname{det}\left(I+X^{2}\right)^{-n+p} d X$ is equal to

$$
\int_{H}\left|\int_{\mathcal{O}_{p}} \mathrm{e}^{i \operatorname{tr}\left(X \xi_{p, 0}\right)} \check{f}\left(\xi_{p}\right) d \mu_{p}\left(\xi_{p}\right) \int_{\mathcal{O}_{n-p}} \mathrm{e}^{i \operatorname{tr}\left[(i I+X) \xi_{n-p}\right]} d \mu_{n-p}\left(\xi_{n-p}\right)\right|^{2} d X .
$$

But Lemma 9.3 allows this to be rewritten as

$$
\int_{H}\left|\int_{\mathcal{O}_{n}}\left(\mathrm{e}^{i \operatorname{tr}(X \cdot)} \check{f}(\cdot) * \mathrm{e}^{i \operatorname{tr}[(i I+X) \cdot]}\right)\left(\xi_{n}\right) d \mu_{n}\left(\xi_{n}\right)\right|^{2} d X .
$$

However, it is easy to check that the definition of $*$ in Lemma 9.3 implies

$$
\left(\mathrm{e}^{i \operatorname{tr}(X \cdot)} \check{f}(\cdot) * \mathrm{e}^{i \operatorname{tr}[(i I+X) \cdot]}\right)\left(\xi_{n}\right)=\mathrm{e}^{i \operatorname{tr}\left(X \xi_{n}\right)}\left(\check{f}(\cdot) * \mathrm{e}^{-\operatorname{tr} \cdot}\right)\left(\xi_{n}\right) .
$$

Thus $\int_{H}|A f(X)|^{2} \operatorname{det}\left(I+X^{2}\right)^{-n+p} d X$ is equal to

$$
\begin{aligned}
& \int_{H}\left|\int_{\mathcal{O}_{n}} \mathrm{e}^{i \operatorname{tr}\left(X \xi_{n}\right)}\left(\check{f}(\cdot) * \mathrm{e}^{-\operatorname{tr} \cdot}\right)\left(\xi_{n}\right) d \mu_{n}\left(\xi_{n}\right)\right|^{2} d X \\
& =\int_{H}\left|\int_{H} \mathrm{e}^{i \operatorname{tr}(X Y)} \chi_{\mathcal{O}_{n}}(Y)\left(\check{f}(\cdot) * \mathrm{e}^{-\operatorname{tr} \cdot}\right)(Y) d Y\right|^{2} d X
\end{aligned}
$$

where $\chi_{\mathcal{O}_{n}}$ is the characteristic function for the open set $\mathcal{O}_{n}$ inside $H$. The next step uses the Plancherel Theorem on the above integral to rewrite it as

$$
\int_{H}\left|\chi_{\mathcal{O}_{n}}(X)\left(\check{f}(\cdot) * \mathrm{e}^{-\operatorname{tr} \cdot}\right)(X)\right|^{2} d X=\int_{\mathcal{O}_{n}}\left|\left(\check{f}(\cdot) * \mathrm{e}^{-\operatorname{tr} \cdot}\right)(X)\right|^{2} d X .
$$


To justify this step, we verify that $\check{f}(\cdot) * \mathrm{e}^{-\operatorname{tr} \cdot} \in L^{1}\left(\mathcal{O}_{n}\right) \cap L^{2}\left(\mathcal{O}_{n}\right)$. By the definition of $*$, we have

$$
\left(\check{f}(\cdot) * \mathrm{e}^{-\operatorname{tr} \cdot)}\left(l \cdot E_{n}\right)=\int_{S_{n} / S_{p} \cap \widetilde{S}_{n-p}} \check{f}\left((l s) \cdot E_{p}\right) \mathrm{e}^{-\operatorname{tr}\left[(l s) \cdot \widetilde{E}_{n-p}\right]} d s .\right.
$$

So

$$
\begin{aligned}
& \int_{\mathcal{O}_{n}} \mid\left(\check{f}(\cdot) * \mathrm{e}^{-\operatorname{tr} \cdot)(X) \mid d X}\right. \\
& =\int_{L / S_{n}}\left|\int_{S_{n} / S_{p} \cap \widetilde{S}_{n-p}} \check{f}\left((l s) \cdot E_{p}\right) \mathrm{e}^{-\operatorname{tr}\left[(l s) \cdot \widetilde{E}_{n-p}\right]} d s\right| d l \\
& \leq \int_{L / S_{n}} \int_{S_{n} / S_{p} \cap \widetilde{S}_{n-p}} \mid \check{f}\left((l s) \cdot E_{p}\right) \mathrm{e}^{-\operatorname{tr}\left[(l s) \cdot \widetilde{E}_{n-p}\right] \mid} d s d l \\
& =\int_{\mathcal{O}_{p} \times \mathcal{O}_{n-p}}\left|\check{f}\left(\xi_{p}\right) \mathrm{e}^{-\operatorname{tr} \xi_{n-p}}\right| d \mu_{p}\left(\xi_{p}\right) d \mu_{n-p}\left(\xi_{n-p}\right)<\infty
\end{aligned}
$$

and so the $L^{1}$ condition follows. For the $L^{2}$ condition, the key observation is that $S_{n} / S_{p} \cap \widetilde{S}_{n-p}$ is compact. In fact, recall $S_{n} / S_{p} \cap \widetilde{S}_{n-p}$ is isomorphic to $U(n) / U(p) \times U(n-p)$. Thus Hölder's inequality can be made use of below to check

$$
\begin{aligned}
& \int_{\mathcal{O}_{n}}\left|\left(\check{f}(\cdot) * \mathrm{e}^{-\operatorname{tr} \cdot}\right)(X)\right|^{2} d X \\
& =\int_{L / S_{n}}\left|\int_{S_{n} / S_{p} \cap \widetilde{S}_{n-p}} \check{f}\left((l s) \cdot E_{p}\right) \mathrm{e}^{-\operatorname{tr}\left[(l s) \cdot \widetilde{E}_{n-p}\right]} d s\right|^{2} d l \\
& \leq \int_{L / S_{n}} k \int_{S_{n} / S_{p} \cap \widetilde{S}_{n-p}} \mid \check{f}\left((l s) \cdot E_{p}\right) \mathrm{e}^{-\left.\operatorname{tr}\left[(l s) \cdot \widetilde{E}_{n-p}\right]\right|^{2}} d s \\
& =k \int_{\mathcal{O}_{p} \times \mathcal{O}_{n-p}}\left|\check{f}\left(\xi_{p}\right) \mathrm{e}^{-\operatorname{tr} \xi_{n-p}}\right|^{2} d \mu_{p}\left(\xi_{p}\right) d \mu_{n-p}\left(\xi_{n-p}\right)<\infty
\end{aligned}
$$

where $k=\operatorname{Vol}\left(S_{n} / S_{p} \cap \widetilde{S}_{n-p}\right)$. Thus the use of the Plancherel Theorem is valid and we may write

$$
\int_{H}|A f(X)|^{2} \operatorname{det}\left(I+X^{2}\right)^{-n+p} d X=\int_{\mathcal{O}_{n}}\left|\left(\check{f}(\cdot) * \mathrm{e}^{-\operatorname{tr} \cdot}\right)(X)\right|^{2} d X .
$$

However, the above calculation that checks the $L^{2}$ condition now implies

$$
\int_{H}|A f(X)|^{2} \operatorname{det}\left(I+X^{2}\right)^{-n+p} d X \leq C \int_{\mathcal{O}_{p}}\left|\check{f}\left(\xi_{p}\right)\right|^{2} d \mu_{p}\left(\xi_{p}\right)=C\|\check{f}\|_{L^{2}\left(\mathcal{O}_{p}\right)}
$$

as desired where $C=k \int_{\mathcal{O}_{n-p}} \mathrm{e}^{-2 \operatorname{tr} \xi_{n-p}} d \mu_{n-p}\left(\xi_{n-p}\right)<\infty$. 


\section{The Main Theorem.}

Theorem 9.1 allows the completion of the maps $A, F_{R}$, and $F_{E}$.

Definition 10.1. Let

$$
A: L^{2}(H)^{+} \rightarrow L^{2}(H)^{-}
$$

be the continuous extension of $A: \mathcal{S}(H) \rightarrow L^{2}(H)^{-}$where we view $\mathcal{S}(H) \subseteq$ $L^{2}(H)^{+}$,

$$
F_{R}: L^{2}(H)^{+} \rightarrow L^{2}\left(\mathcal{O}_{p}\right)
$$

be the continuous extension of $F_{R}: \mathcal{S}(H) \rightarrow L^{2}\left(\mathcal{O}_{p}\right)$, and

$$
F_{E}: \operatorname{Im}\left(F_{R}\right) \rightarrow L^{2}(H)^{-}
$$

be the continuous extension of $F_{E}: \operatorname{Im}\left(\left.F_{R}\right|_{\mathcal{S}(H)}\right) \rightarrow L^{2}(H)^{+}$where $\operatorname{Im}\left(F_{R}\right)$ denotes the image of $F_{R}$ on $L^{2}(H)^{+}$.

Two notes are in order. The first is that Definition 7.2 already gives a definition of $A=B S$ on all of $\mathcal{S}(H)^{+}$which Theorem 7.2 shows is well defined. However, it is apriori possible (though not true) that Definition 10.1 defines $A$ differently on $\mathcal{S}(H)^{+} \backslash \mathcal{S}(H)$. This ambiguity is removed in Lemma 10.1 below.

The second note is that the closure of $\operatorname{Im}\left(F_{R}\right)$ is in fact all of $L^{2}\left(\mathcal{O}_{p}\right)$. This is shown in Theorem 10.4 below.

Lemma 10.1. When restricted to $A: \mathcal{S}(H)^{+} \rightarrow L^{2}(H)^{+}$, both Definitions 7.2 and 10.1 coincide.

Proof. We break the proof of this Lemma up into steps. In this proof only, write $\bar{A}$ for the operator defined in Definition 10.1 by extending continuously from $\mathcal{S}(H)$ to $\mathcal{S}(H)^{+}$with respect to the $L^{2}(H)^{+}$and $L^{2}\left(\mathcal{O}_{p}\right)$ norms, respectively. Likewise for this proof only, write $A$ for the operator defined in Definition 7.2 as $B \circ S$ on $\mathcal{S}(H)^{+}$(Theorem 7.2 shows it is well defined).

(1). The first step is found in [14], Lemma 1. Since the proof is straightforward and identical to the one in [14], we simply state the result. Namely, $f \in C^{\infty}(H)$ has a (unique) smooth extension via the open dense embedding $H \cong \bar{N} \hookrightarrow G / P$ to a function in the smooth principal series $I_{p}^{ \pm}$ if and only if the function

$$
X \rightarrow(g f)(X),
$$

initially defined for $X \in H$ with $\operatorname{det}(D-X B) \neq 0$ by Theorem 5.5 , extends to a smooth function on $H$ for each $g=\left(\begin{array}{cc}A & B \\ C & D\end{array}\right) \in G$.

(2). Suppose $X_{0} \in H$ and $\operatorname{det}\left(A+B X_{0}\right)=0$. Choose $X_{t}, t \in \mathbb{R}$, a smooth path in $H$ with $\operatorname{det}(D-X(t) B)$ not identically zero as a function of t. Let $\|\cdot\|$ be a norm on $H$. Then $\lim _{t \rightarrow 0}\left\|g X_{t}\right\|=\infty$. This follows by 
the definition of the linear fractional action in Definition 4.1 combined with expressing the inverse of a matrix in terms of cofactors and its determinant.

(3). Steps (1) and (2) may now be combined with Theorem 5.5 (see also Definition 4.1 and Equation (2.3)) to show that each $\phi \in \mathcal{S}(H)$ has a smooth extension to $I_{p}^{+}$. In particular, the smooth extension of $X \rightarrow(g \phi)(X)$ is the map sending $X$ to

$$
\operatorname{det}\left(D^{*}-B^{*} X\right)^{-2 n+p} \phi\left(\left(-C^{*}+A^{*} X\right) /\left(D^{*}-B^{*} X\right)^{-1}\right)
$$

if $\operatorname{det}\left(D^{*}-B^{*} X\right) \neq 0$ and sending $X$ to 0 if $\operatorname{det}\left(D^{*}-B^{*} X\right)=0$. By $G$ invariance of $I_{p}^{+}$, we also conclude that each function in $\mathcal{S}(H)^{+}$has a smooth extension to $I_{p}^{+}$. Moreover using (1), it is easy to check that if $\psi \in I_{p}^{+}$is the extension of an element in $\mathcal{S}(H)^{+}$, then $\psi$ is identically zero on all points of $G / P$ in the compliment of $\bar{P}$.

(4). Each $\psi \in I_{p}^{+}$is bounded and when restricted to $H$ satisfies the growth condition $|\psi(X)| \leq C\|X\|^{-2 n+p}$ for some constant $C$. This follows easily from Equation (5.1) and unitary diagonalization of $X$.

(5). Fix $\psi \in \mathcal{S}(H)^{+}$. Also denote by $\psi$ its smooth extension to $G / P$. For $r>0$ choose cut-off functions $\phi_{r} \in C_{0}^{\infty}(H)$ with range in $[0,1]$ so that $\phi_{r}$ is identically 1 on the ball of radius $r$ about the origin and identically 0 outside the ball of radius $r+1$. Then the fact that $\psi \in L^{2}(H)^{+}$and points (3) and (4) show that as $r \rightarrow \infty$ that $\phi_{r} \psi \rightarrow \psi$ in the $L^{2}$-norm and that $\phi_{r} \psi \rightarrow \psi$ uniformly as functions on either $H$ or $G / P$.

(6). $S: I_{p}^{+} \rightarrow C^{\infty}(G / K)$ is continuous in the smooth topology of uniform convergence on compact sets. This follows since $S$ is an integral of smooth functions over a compact set (Definition 3.2).

(7). Suppose that $f, f_{i} \in \mathcal{S}(H)^{+}$so that $f_{i} \rightarrow f$ uniformly. Then $A f_{i} \rightarrow A f$ pointwise. To see this, use the definition of $A$ in the first step below, uniform convergence in the second, and point (6) in the third to calculate

$$
\begin{aligned}
\lim _{i \rightarrow \infty} A f_{i}(X) & =\lim _{i \rightarrow \infty} \lim _{\eta \in \mathcal{D}^{+}, \eta \rightarrow X} S f_{i}(X) \\
& =\lim _{\eta \in \mathcal{D}^{+}, \eta \rightarrow X} \lim _{i \rightarrow \infty} S f_{i}(X) \\
& =\lim _{\eta \in \mathcal{D}^{+}, \eta \rightarrow X} S f(X) \\
& =A f(X) .
\end{aligned}
$$

(8). We now prove the Lemma. Let $\psi \in \mathcal{S}(H)^{+}$and pick $\phi_{r}$ as in point (5). Note that $\phi_{r} \in \mathcal{S}(H)$. Thus, by definition,

$$
\bar{A} \psi=\lim _{r \rightarrow \infty} A \phi_{r} \psi
$$

in the $L^{2}$-sense. We may therefore choose a subsequence so that $\bar{A} \psi=$ $\lim _{r \rightarrow \infty} A \phi_{r} \psi$ pointwise almost everywhere. But points (5) and (7) imply 
that $\lim _{r \rightarrow \infty} A \phi_{r} \psi=A \psi$ pointwise everywhere. In particular, we see $\bar{A} \psi=$ $A \psi$ almost everywhere so that $\bar{A}=A$ in the $L^{2}$-sense on $\mathcal{S}(H)^{+}$.

We now show that $A$ remains a $G$ map.

Theorem 10.2. The map

$$
A: L^{2}(H)^{+} \rightarrow L^{2}(H)^{-}
$$

is a $G$ map and $A=F_{R} \circ F_{E}$.

Proof. It follows from Lemma 10.1 and Theorem 7.2 that $A$ is a $G$-map on $\mathcal{S}(H)^{+}$. Theorem 7.2 also shows that $A=F_{R} \circ F_{E}$ on $\mathcal{S}(H)$. The denseness of $\mathcal{S}(H)$ and a continuity argument suffice to finish the proof of this Theorem.

We are able to complete Definition 8.2 and Theorem 8.1 as follows.

Definition 10.2. If $f_{1}, f_{2} \in L^{2}(H)^{+}$, let

$$
\left\langle f_{1}, f_{2}\right\rangle_{A}=i^{n p} \int_{H} f_{1}(X) \overline{A f_{2}(X)} d X .
$$

By Hölder's inequality (multiply by the det and its inverse), the form $\left\langle f_{1}, f_{2}\right\rangle_{A}$ is well defined and bounded by the product of the norm of $f_{1} \in$ $L^{2}(H)^{+}$and the norm of $A f_{2} \in L^{2}(H)^{-}$. Theorem 8.1 and continuity imply the following.

Theorem 10.3. If $f_{1}, f_{2} \in L^{2}(H)^{+}$, then

$$
\left\langle f_{1}, f_{2}\right\rangle_{A}=\left\langle F_{R} f_{1}, F_{R} f_{2}\right\rangle_{\mathcal{O}_{p}} .
$$

Moreover, the form $\langle\cdot, \cdot\rangle_{A}$ is $G$-invariant.

This suggests that we try to make $L^{2}\left(\mathcal{O}_{p}\right)$ into a $G$-space in such a way that $F_{R}$ is a $G$-map. In turn, this Theorem 10.3 ought to induce a $G$ invariant structure on a quotient of the principal series. First observe that $F_{E}$ is injective (for instance, by the Stone-Weierstrass theorem and the fact that the characters $\mathrm{e}^{i \operatorname{tr}(\xi \cdot)}$ separate points). This implies that

$$
\operatorname{ker}(A)=\operatorname{ker}\left(F_{R}\right)
$$

and in particular that $\operatorname{ker}\left(F_{R}\right)$ is $G$-invariant. Thus there is a $G$-action on

$$
L^{2}(H)^{+} / \operatorname{ker}\left(F_{R}\right)
$$

for which $\langle\cdot, \cdot\rangle_{A}$ descends to a $G$-invariant, positive (by Theorem 10.3), Hermitian two-form. Moreover, the following is well defined.

Definition 10.3. For $g \in G, f \in L^{2}(H)^{+}$, and $h=F_{R} f \in L^{2}\left(\mathcal{O}_{p}\right)$, define

$$
g(h)=F_{R}(g f)
$$

or equivalently

$$
g h=F_{E}^{-1}\left(g F_{E} h\right)
$$


This equivalence is trivial to check using Theorem 10.2. This definition makes $\operatorname{Im}\left(F_{R}\right) \subseteq L^{2}\left(\mathcal{O}_{p}\right)$ into a representation of $G$ so that both $F_{R}$ and $F_{E}$ are now $G$-maps. In general, we have to take closures to complete the picture.

Definition 10.4. Let $\mathcal{K}=\left\{f \in L^{2}(H)^{+} \mid\langle f, f\rangle_{A}=0\right\}=\operatorname{ker}\left(F_{R}\right)=$ $\operatorname{ker}(A)=\left\{f \in L^{2}(H)^{+} \mid\langle f, g\rangle_{A}=0, \forall g \in L^{2}(H)^{+}\right\}$. Let

$$
\overline{L^{2}(H)^{+} / \mathcal{K}}
$$

be the completion of $L^{2}(H)^{+} / \mathcal{K}$ with respect to $\langle\cdot, \cdot\rangle_{A}$.

We use continuity to extend the $G$-action to all of $\overline{L^{2}(H)^{+} / \mathcal{K}}$, continuity to extend the map

$$
F_{R}: \overline{L^{2}(H)^{+} / \mathcal{K}} \rightarrow L^{2}\left(\mathcal{O}_{p}\right),
$$

and the fact that $A=F_{E} \circ F_{R}$ to extend the map

$$
A: \overline{L^{2}(H)^{+} / \mathcal{K}} \rightarrow L^{2}(H)^{-} \text {. }
$$

Then we have the following.

Theorem 10.4. $\operatorname{Im}\left(F_{R}\right)=L^{2}\left(\mathcal{O}_{p}\right)$. Moreover, $L^{2}\left(\mathcal{O}_{p}\right)$ is an irreducible unitary representation of $G$ that, in fact, remains irreducible under restriction to $\bar{P}$.

Proof. Unitarity follows immediately from Theorem 10.3. The rest of the argument is entirely classical. Let $I=\operatorname{Im}\left(F_{R}\right)$. Recall that $F_{R}$ is an isometry mapping the closure of $L^{2}(H)^{+} / \mathcal{K}$ into $L^{2}\left(\mathcal{O}_{p}\right) . \quad I$ is therefore a closed, non-trivial subspace of $L^{2}\left(\mathcal{O}_{p}\right)$ since, in particular, $\check{\phi}_{\left.\right|_{\mathcal{O}_{p}}} \in I$ for each $\phi \in \mathcal{S}(H)$. It is easy to check (Definitions 10.3 and 8.1, Theorem 5.5, and Equation (5.3)) that

$$
\begin{aligned}
\left(\begin{array}{ll}
I & 0 \\
X & I
\end{array}\right) f(\xi) & =e^{-i \operatorname{tr}(X \xi)} f(\xi) \\
\left(\begin{array}{ll}
A & 0 \\
0 & A^{*-1}
\end{array}\right) f(\xi) & =\operatorname{det}(A)^{-p} f\left(A^{-1} \xi A^{*-1}\right)
\end{aligned}
$$

for each $f \in I$. We can use the same formulas above to extend the action of $\bar{P}$ on $I$ to an action on all of $L^{2}\left(\mathcal{O}_{p}\right)$. It is a fact that this action is irreducible. We sketch the idea (see [16] $\S 1.2$, Theorem 2.1 or [13] $\S 3.1)$. It suffices to show that any bounded intertwining operator, $T$, is a constant. However, it can be shown that commuting with $\bar{N}$ implies that $T$ is multiplication by a bounded function. The transitivity of the $L$ action then implies that $T$ is a constant. Thus $L^{2}\left(\mathcal{O}_{p}\right)$ is irreducible under $\bar{P}$. Since $I$ is $\bar{P}$-invariant, this implies that $I=L^{2}\left(\mathcal{O}_{p}\right)$ which finishes the proof. 
This Theorem induces a unitary structure on a quotient of the principal series.

Corollary 10.5. $\langle\cdot, \cdot\rangle_{A}$ induces a $G$-invariant unitary structure on the quotient space $\overline{L^{2}(H)^{+} / \mathcal{K}}$ that is unitarily isomorphic to $L^{2}\left(\mathcal{O}_{p}\right)$ by $F_{R}$. In particular,

$$
\left\langle f_{1}, f_{2}\right\rangle_{A}=\left\langle F_{R} f_{1}, F_{R} f_{2}\right\rangle_{\mathcal{O}_{p}} .
$$

for all $f_{1}, f_{2} \in L^{2}(H)^{+}$.

Proof. This is contained in Theorem 10.3, 10.4, and Equation (10.1).

This completes diagram 8.2 to the following diagram of unitary $G$-maps where $F_{R}$ is an isomorphism and $A$ (viewed as a map on the quotient space) and $F_{E}$ are injective:

$$
\begin{array}{cccc}
\frac{A}{L^{2}(H)^{+} / \mathcal{K}} & \underset{1}{\longrightarrow} & L^{2}(H)^{-} & \\
& F_{R} & \uparrow & F_{E} . \\
& & L^{2}\left(\mathcal{O}_{p}\right) &
\end{array}
$$

This Diagram then fits into a larger diagram that incorporates Diagram 7.4. Namely, define $F_{\widetilde{E}}: L^{2}\left(\mathcal{O}_{p}\right) \rightarrow C^{\infty}\left(\mathcal{D}^{+}\right)$by

$$
F_{\widetilde{E}} f(\eta)=i^{n p} \int_{\mathcal{O}_{p}} \mathrm{e}^{i \operatorname{tr} \eta \xi} f(\xi) d \xi
$$

which is well defined by Hölder's inequality. It is immediate that we obtain a commuting diagram of $G$-maps,

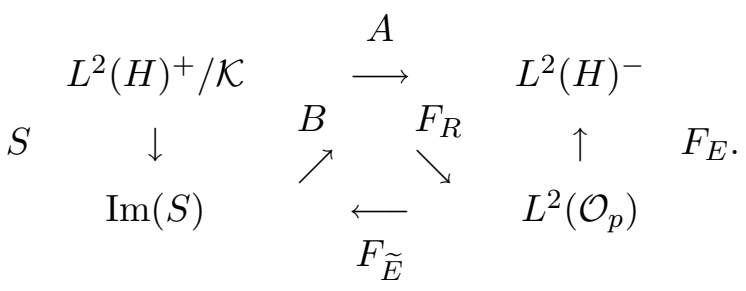

Also note that the injectivity of $F_{\widetilde{E}}$ is enough to strengthen Corollary 10.5 so that $B$ is injective and

$$
\mathcal{K}=\operatorname{ker}(A)=\operatorname{ker}(S)=\operatorname{ker}\left(F_{R}\right)=\operatorname{ker}\left(\langle\cdot, \cdot\rangle_{A}\right) .
$$




\section{References}

[1] L. Barchini, Szegö kernels associated with Zuckerman modules, J. Funct. Anal., 131 (1995), 145-182.

[2] L. Barchini, S. Gindikin and H.-W. Wong, The geometry of the flag manifold and holomorphic extension of Szegö kernels for $U(p, q)$, Pacific J. Math., to appear.

[3] L. Barchini, A. Knapp and R. Zierau, Intertwining operators into Dolbeault cohomology representations, J. Funct. Anal., 107 (1992), 302-341.

[4] J. Faraut and A. Korányi, Analysis on Symmetric Cones, Clarendon Press, Oxford, 1994.

[5] Harish-Chandra, Representations of a semisimple Lie group on a Banach space I, Trans. Amer. Math. Soc., 70 (1953), 185-243.

[6] S. Helgason, Groups and Geometric Analysis: Integral Geometry, Invariant Differential Operators, and Spherical Functions, Academic Press, 1984.

[7] L.K. Hua, Harmonic Analysis of Functions of Several Complex Variables in the Classical Domains, American Mathematical Society, Providence, 1963.

[8] M. Kashiwara and M. Vergne, Functions on the Shilov boundary of the generalized half plane, Springer-Verlag Lecture Notes in Math., 728 (1979), 136-176.

[9] A. Knapp, Representation Theory of Semisimple Groups: An Overview Based on Examples, Princeton University Press, Princeton, 1986.

[10] A. Knapp and K. Okamoto, Limits of holomorphic discrete series, J. Funct. Anal., 9 (1972), 375-409.

[11] A. Knapp and N. Wallach, Szegö kernels associated with discrete series, Invent. Math., 34 (1976), 163-200.

[12] H. Rossi and M. Vergne, Analytic continuation of the holomorphic discrete series of a semi-simple Lie group, Acta Math., 136 (1976), 1-59.

[13] S. Sahi, Explicit Hilbert spaces for certain unipotent representations, Invent. Math., 110 (1992), 409-418.

[14] S. Sahi and E. Stein, Analysis in matrix space and Speh's representations, Invent. Math., 101 (1990), 379-393.

[15] E. Stein and T. Murphy, Harmonic Analysis: Real-Variable Methods, Orthogonality, and Oscillatory Integrals, (Princeton Mathematical, No 43), Princeton University Press, Princeton, 1993.

[16] M. Taylor, Noncommutative Harmonic Analysis, Mathematical Surveys and Monographs No. 22, American Mathematical Society, Providence, RI 1946.

Received August 20, 1999 and revised February 24, 2000. The authors were partially supported by the National Science Foundation under Grant No. DMS 9801605 and DMS 9623280, respectively. 
E-mail address: leticia@math.okstate.edu

Department of Mathematics

BAYLOR UNIVERSITY

PO Box 97328

WACO, TX 76798-7328

E-mail address: Mark_Sepanski@Baylor.edu 